\title{
Fibroepithelial Stromal Polyp as Low Back Lesion in 88-year-old Man: a Case Report
}

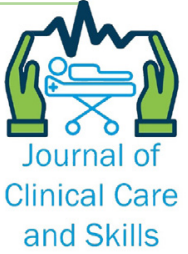

and Skills

\section{ARTICLE INFO}

\section{Article Type}

Case Report

\section{Authors}

Daneshyar Z. ${ }^{1} M D$,

Shokripour M. ${ }^{* 2} M D$

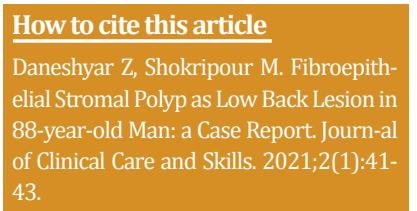

${ }^{1}$ Clinical Research Development Unit, Yasuj University of Medical Sciences, Yasuj, Iran 2Department of Pathology, Medical School, Shiraz University of Medical Sciences, Shiraz, Iran

\section{*Correspondence}

Address: Department of Pathology, Medical School, Shiraz University of Medical Sciences, Shiraz, Iran. Postal Code: 7183833387

Phone: -

Fax: +98 (71) 32301784

shokripurm@sums.ac.ir

\section{Article History}

Received: May 20, 2020

Accepted: August 08, 2020

ePublished: February 01, 2021

\begin{abstract}
A B S T R A C T
Introduction Fibroepithelial stromal polyp (FESP) of the female lower genital tract is a rare benign lesion characterized by polypoid proliferation of stroma, covered by a benign squamous epithelium in the vulvovaginal region. These hormone-sensitive lesions usually occur in young to middle-aged women. The lesions are typically no larger than $5 \mathrm{~cm}$ in diameter and are found randomly during routine gynecologic examinations.

Patient Information The case of this study was an 88-year-old man with a solitary polypoid lesion measuring $3 \times 2 \times 2$ in the low back area showing the histological appearances of those described in the vulvovaginal region.

It was a well-circumscribed gray-brown lesion covered by wrinkled skin. Histopathological examination revealed edematous hypocellular lesion at low magnification with hyperplastic squamous epithelium coating with reactive epithelial change. At high magnification, the lesion consisted of bland-appearing spindle and stellate-shaped cells in an edematous and fibrous stroma with a large number of variably-sized blood vessels. Immunohistochemistry showed the lesional cells to be focally immunoreactive for EMA and negative for CD34, SMA in stromal cells.

Conclusion FESPs is an uncommon benign lesion that can occur in men and areas other than the genitals with independence from female sex hormones. Although this is a benign finding, reporting it will increase the knowledge about this rare tumor.
\end{abstract}

Keywords Polyp; Skin; Back 


\section{Introduction}

Fibroepithelial stromal polyp (FEP), also known as a mesodermal stromal polyp, is a rare benign polypoid subepithelial mesenchymal proliferation of the lower female genital tract characterized by polypoid proliferation of stroma [1], covered by a benign squamous epithelium [2]. It is a site-specific mesenchymal neoplasm of the vulvovaginal region [3] present at the vagina, vulva, or cervix [1]. More commonly, in the vulva, and rarely are found in extragenital sites ${ }^{[4]}$.

These lesions usually occur in young to middle-aged women, especially the obese ones [5]. These polyps are hormone-sensitive and most commonly occur in pregnancy. However, they can also be seen in premenopausal females who are on hormone replacement therapy [6].

These lesions typically do not grow larger than $5-\mathrm{cm}$ in diameter mostly. The lesions' size is $1 \times 2 \mathrm{~cm}{ }^{[7]}$ and is found incidentally during routine gynecologic exams ${ }^{[8]}$. Here, we present a case of a fibroepithelial stromal polyp arising from the low back of an 88year-old male, showing histological appearances of those described in the vulvovaginal region unusual manifestation of this disease.

\section{Patient Information}

An 88-year-old man with chronic obstructive pulmonary disease (COPD) and hypertension was hospitalized in ICU due to pneumonia and respiratory distress. The surgeon found a solitary polypoid lesion measuring $3 \times 2 \times 2 \mathrm{~cm}$ in the low back area without any other similar lesions in his body during the examination. The lesion had been appeared as a small nodule about a long time ago and grew up gradually at this time. It was not the chief complaint of the patient. No radiographic imaging was done for the patient before surgery. The patient's drug history indicated antihypertensive drugs, and steroid medication was not seen in their medical history. The patient was treated by local excision of the lesion under general anesthesia. No further local treatment was given.

Grossly the mass was well-circumscribed graybrown and covered by wrinkled but otherwise unremarkable skin.

Histopathological examination revealed edematous hypocellular lesion at low magnification with hyperplastic squamous epithelium coating with reactive epithelial change; underlying subepithelial tissue composed of loose collagenous tissue. There was no demarcation between the lesion and the epidermis.

At high magnification, the lesion consisted of blandappearing spindle and stellate-shaped cells in an edematous and fibrous stroma with scattered chronic inflammatory cells. A large number of variably-sized blood vessels were seen. There was no evidence of necrosis and mitosis (Figure 1).

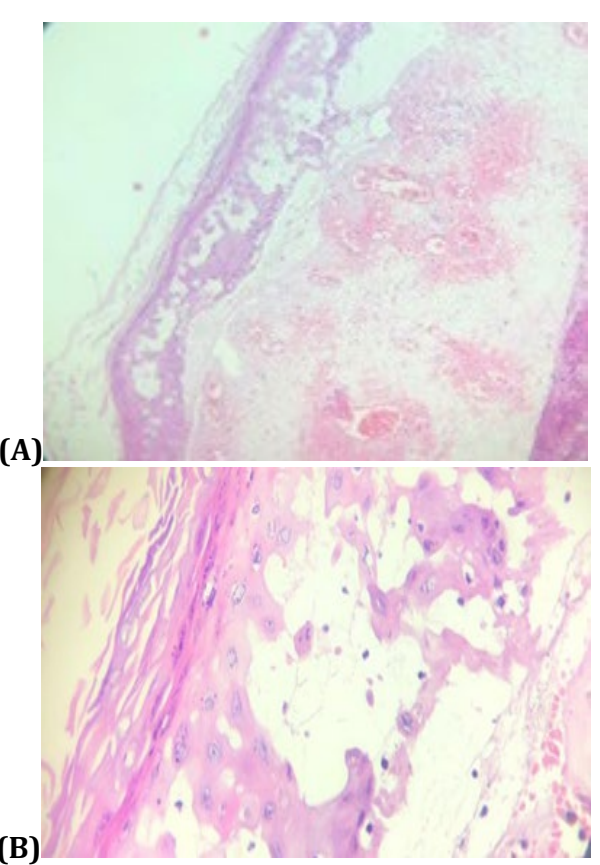

Figure 1) Histological appearances of the lesion. A: Rather hypocellular edematous stroma and increased number of blood vessels, B: Overlying epithelium with reactive epithelial change

(A)

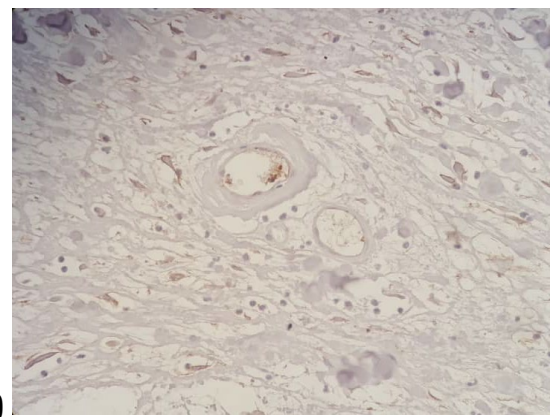

(B)

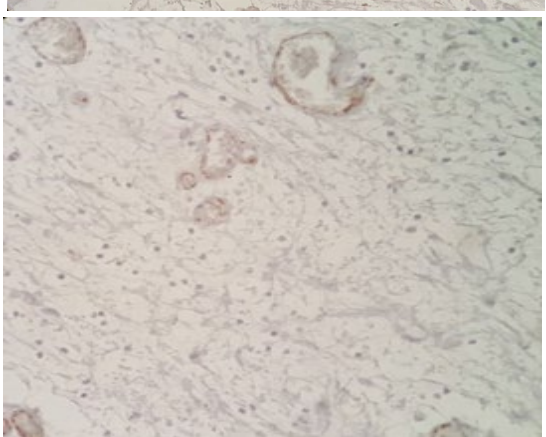

(C)

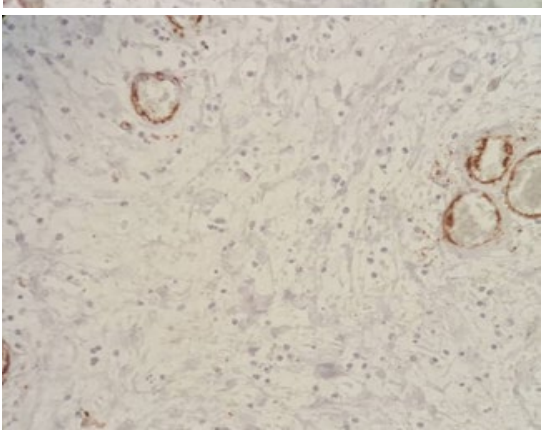

Figure 2) Immunohistochemical finding in Stromal cells A: EMA weakly positive, B: SMA negative, C: CD34 negative 
No plasmacytoid cells, thick-walled vessels, or cells with epithelioid morphology were observed.

Immunohistochemistry showed the lesional cells to be focally immunoreactive for EMA and negative for CD34, SMA in stromal cells (Figure 2).

\section{Discussion}

The fibroepithelial polyp is a very common benign tumor of the skin [9]. They commonly occur on the neck, axilla, perineum, and thighs. The fibroepithelial stromal polyp (FESP) are infrequent mesenchymal lesions of the distal female genital tract found in the vulvovaginal region [10].

The reported lesions' morphological features are fully in keeping with a benign lesion, as its name says. In this context, it is important to differentiate this from a more common benign skin lesion of adults, fibroepithelial polyp. Fibroepithelial stromal polyps are polypoid but usually contain the hypocellular stroma consisting of bland-appearing spindle cells in an edematous stroma hyperplastic squamous epithelium with reactive epithelial change. Fibroepithelial polyp histologically shown fibrovascular cores covered by squamous epithelium, the central core composed of loose collagen. Nevertheless, they do not show edematous and myxoid stroma, nor they show typical reactive atypia, which we saw in our case. The other differential diagnosis is spindle cell metaplastic carcinoma-which can appear deceptively bland. This current case shows no expression of epithelial markers. (EMA negative) in comparison with spindle cell metaplastic carcinoma, which is EMA positive. other possible differentials are pleomorphic fibroma that has atypical single fibroblasts which are CD34 positive [11] that our case was CD34 negative

In this case, an 88year-old man presents with a large size fibroepithelial stromal polyp in the low back area. The low back compartment is an unusual sight for the origin of a fibroepithelial stromal polyp Second and third unusual thing about this lesion was that the lesions mostly occur in middle-aged women. Our case was a man in his old age. The fourth unusual thing was its feminine sex Hormone Independence in our case. While fibroepithelial stromal polyp of Vulva exhibit hormonal responsiveness and female hormones influence their growth ${ }^{[11]}$ In the best of our knowledge, except for 2 cases of large fibroepithelial stromal polyp presenting as a nipple mass reported by Shaaban et al. and Rexhepi et al. no recently published that was reported such a similar extragenital presentation ${ }^{[5,7]}$.

\section{Conclusion}

FESPs is an uncommon benign lesion that can occur in men and areas other than the genitals with
Daneshyar Z. \& Shokripour M.

independence from female sex hormones. Although this is a benign finding, reporting it will increase the knowledge about this rare tumor.

Acknowledgments: We are over helmed in all humbleness and gratefulness to acknowledge my depth to all those who have helped me to put these ideas, Mr. Aiin, and Mr. Taghavi Pathology technicians and the other Partners

Ethical Permissions: Not declared.

Conflict of Interests: There is no conflict of interest.

Authors' Contributions: Daneshyar Z. (First author), Introduction author/Methodologist/Original researcher/Discussion author (60\%); Shokripour M. (Second author), Introduction author/Methodologist/Discussion author (40\%)

Funding/Sources: There was no source of funding.

\section{References}

1- Yoo J, Je BK, Yeom SK, Park YS, Min KJ, Lee JH. Giant fibroepithelial stromal polyp of the vulva: Diffusionweighted and conventional magnetic resonance imaging features and pathologic correlation. J Pediatr Adolesc Gynecol. 2019;32(1):93-7.

2- Halvorsen TB, Johannesen E. Fibroepithelial polyps of the vagina: Are they old granulation tissue polyps? J Clin Pathol. 1992;45(3):235-40.

3- Bozgeyik Z, Kocakoc E, Koc M, Ferda Dagli A. Giant fibroepithelial stromal polyp of the vulva: Extended fieldof-view ultrasound and computed tomographic findings. Ultrasound Obstet Gynecol. 2007;30(5):791-2.

4- Zulfikaroglu E, Kilic S, Taflan S, Tarhan I, Akbay S, Mollamahmutoglu L. Vulvar fibroepithelial stromal polyp in the early pregnancy. Van Tip Dergisil. 2013;20(2):88-90. [Turkish]

5- Shaaban AM, Turton EP, Merchant W. An unusual case of a large fibroepithelial stromal polyp presenting as a nipple mass. BMC Res Notes. 2013;6:345.

6- Lee MH, Hwang JY, Lee JH, Kim DH, Song SH. Fibroepithelial polyp of the vulva accompanied by lymphangioma circumscriptum. Obstet Gynecol Sci. 2017;60(4):401-4.

7- Rexhepi M, Trajkovska E, Besimi F, Rufati N. Giant fibroepithelial polyp of vulva: A case report and review of literature. Prilozi. 2018;39(2-3):127-30.

8- Madueke-Laveaux OS, Gogoi R, Stoner G. Giant fibroepithelial stromal polyp of the vulva: Largest case reported. Ann Surg Innov Res. 2013;7:8.

9- Arora BK. A clinical case of large fibroepithelial polyp of breast nipple. Int J Case Rep Images. 2019; 10:100997Z01BA2019.

10- Hasan M, Afrose R, Kamal M. Cellular pseudosarcomatous fibroepithelial stromal polyp of the cervix: A lesion mimicking as sarcoma. Adv Cytol Pathol. 2018;3(1):21-3.

11- Elwood HR. Skin nonmelanocytic tumor fibrous, fibrohistiocytic and myofibroblastic neoplasms fibroepithelial polyp [Internet]. Detroit: Pathology Outlines; 2015 [Cited 20209 29]. Available from: https://www.pathologyoutlines.com/topic/skintumornon melanocyticfibroepithelialpolyp.html. 